\title{
Análise quantitativa da vegetação lenhosa da Caatinga em Teixeira, PB
}

José Adelmo Nunes Leite ${ }^{1}$, Lúcio Valério Coutinho de Araújo ${ }^{1}$, Eder Ferreira Arriel ${ }^{1 *}$, Lúcia de Fátima de Carvalho Chaves², Assíria Maria Ferreira da Nóbrega ${ }^{1}$

${ }^{1}$ Universidade Federal de Campina Grande, Unidade Acadêmica de Engenharia Florestal, Campus de Patos, CP 61, CEP 58.708-110, Patos, PB, Brasil, ${ }^{2}$ Universidade Federal Rural de Pernambuco, Departamento de Ciências Florestais, Rua Dom Manoel de Medeiros, s/n, Dois Irmãos, CEP: 52171-900, Recife, PE, Brasil

\author{
"Autor correspondente: \\ earriel@gmail.com \\ Termos para indexação: \\ Caracterização florística \\ Antropismo \\ Inventário florestal \\ Index terms: \\ Floristic characterization \\ Anthropogenic \\ Forest inventory
}

Histórico do artigo:

Recebido em 05/09/2013

Aprovado em 03/06/2015

Publicado em 30/06/2015

doi: 10.4336/2015.pfb.35.82.584
Resumo - A cobertura vegetal da região semiárida do Nordeste brasileiro é compreendida pela caatinga, que é, em grande parte, determinada pelo clima e relevo. A região do município de Teixeira, $\mathrm{PB}$ apresenta uma cobertura vegetal de caatinga de porte e densidade diferente das encontradas na Depressão Sertaneja, motivada por maior umidade. O objetivo deste trabalho foi quantificar os remanescentes florestais do município de Teixeira, PB, por meio de técnicas de geoprocessamento e inventário florestal. Para o mapeamento da vegetação nativa, foram utilizadas imagens de satélites, referentes ao mês de novembro de 2008. Foi realizado inventário florestal com uso de amostragem por parcelas, selecionadas sistematicamente, em um total de 40 unidades amostrais com dimensões de $20 \mathrm{~m}$ x $20 \mathrm{~m}$. Foi medida a altura e diâmetro a $1,30 \mathrm{~m}$ do solo (DAP) de todas as árvores com DAP $\geq 10 \mathrm{~cm}$. Cada árvore foi identificada pelo seu nome popular e científico. A vegetação arbórea amostrada foi de 4.911 indivíduos, pertencentes a 46 espécies e 24 famílias, o que corresponde a 3.069 indivíduos ha-1 . As espécies com maior valor de importância (VI) foram: Croton sonderianus, Mimosa tenuiflora e Piptadenia stipulacea. As famílias que mais se destacaram foram Euphorbiaceae, Mimosaceae e Caesalpinaceae. O município de Teixeira apresenta áreas mais preservadas nas regiões serranas, de difícil acesso.

\section{Quantitative analysis of savanna wood species, in Teixeira, state of Paraiba, Brazil}

\begin{abstract}
Savanna is the vegetation of semi-arid northeastern region of Brazil, which is largely determined by climate and topography. The municipality of Teixeira in the state of Paraiba presents hyperxerophilous shrub-arboreal caatinga land cover, which differs in size and density from those found in Sertaneja Depression, due to higher humidity. The objective of this work was to quantify forest remnant in the municipality of Teixeira, using geographic information system and forest inventory techniques. Native vegetation was mapped using satellite images, from November, 2008. Forest inventory was carried out in 40 sample plots $(20 \mathrm{~m} \times 20 \mathrm{~m})$ that were selected systematically. Height and diameter at $1.30 \mathrm{~m}$ from soil level $(\mathrm{DBH})$ were measured in every tree with $\mathrm{DBH} \geq 10$ $\mathrm{cm}$. Each tree was identified by common and scientific names. A total of 4,911 trees were sampled, representing 46 species and 24 families that corresponds to 3,069 trees $\mathrm{ha}^{-1}$. The species with highest importance value (IV) were: Croton sonderianus, Mimosa tenuiflora and Piptadenia stipulacea and the more important families were Euphorbiaceae, Mimosaceae and Caesalpinaceae. The municipality of Teixeira has its best preserved areas in mountainous regions with difficult access.
\end{abstract}




\section{Introdução}

Caatinga é o bioma predominante na região Nordeste do Brasil, cobrindo $54,53 \%$ de sua área total (Santana \& Souto, 2006), sendo o maior e mais importante bioma dessa região, cuja vegetação é conhecida como mata seca ou mata branca (Araújo, 2007). Essa vegetação, que ocupa cerca de $11 \%$ do território brasileiro (Andrade et al., 2005), é xerófila, de fisionomia e florística variada (Ramalho et al., 2009), sendo formada por um mosaico de arbustos espinhosos e florestas sazonais secas, que cobre a maior parte dos estados do Piauí, Ceará, Rio Grande do Norte, Paraíba, Pernambuco, Alagoas, Sergipe, Bahia e o nordeste de Minas Gerais (Leal et al., 2005), de clima semiárido (Maia, 2004).

O semiárido do nordeste brasileiro ocupa $788.064 \mathrm{~km}^{2}$ e se caracteriza por precipitações erráticas no tempo e no espaço, especialmente em áreas mais secas (Rodal et al., 2003). Segundo Fabricante \& Andrade (2007), o bioma Caatinga é considerado uma das 37 grandes regiões geográficas do planeta, possuindo a vegetação mais heterogênea dentre os biomas brasileiros. É considerado muito importante do ponto de vista biológico, por ser um dos poucos que tem ocorrência restrita ao Brasil, apresentando fauna e flora únicas, formadas por uma vasta biodiversidade (Franca-Rocha et al., 2007). Porém, apesar de ser um bioma rico em número de espécies, é floristicamente pobre, em comparação com florestas tropicais úmidas (Monteiro et al., 2015).

De acordo com Rodal et al. (2008), sua variada cobertura vegetal está, em grande parte, determinada pelo clima, relevo e embasamento geológico que, em suas múltiplas interrelações, resultam em ambientes ecológicos bastante variados e se traduz em diferentes paisagens (Santana \& Souto, 2006), como os vales úmidos, as chapadas sedimentares e as amplas superfícies pediplanadas.

Para Leal et al. (2007), sua formação vegetal apresenta características adaptativas bem definidas: árvores baixas e arbustos que, em geral, perdem as folhas na estação das secas (espécies caducifólias), além de muitas cactáceas. Em termos de vegetação brasileira, a classificação hierárquica ainda não está completamente delineada (Alcoforado-Filho et al., 2003). Em relação ao meio ambiente, os maiores problemas associados ao semiárido são o elevado grau de degradação ambiental e o baixo conhecimento quantitativo e qualitativo de sua biodiversidade. Porém, levantamentos florísticos na caatinga indicam que estas áreas apresentam uma extraordinária diversidade e um grande número de espécies endêmicas (Ramalho et al., 2009).

Sendo assim, algumas das linhas de pesquisa que devem ser priorizadas são aquelas voltadas para um melhor conhecimento da biodiversidade e do seu uso pelas populações locais (Araújo, 2007). Porém, Rodal et al. (2008) afirmam que a maioria dos trabalhos não define de forma clara a questão do grau de perturbação das variações fisionômicas dessa vegetação.

Desse modo, Araújo (2007) destaca que é necessário continuar desenvolvendo levantamentos que possam identificar todas as espécies da caatinga, seus padrões de distribuição, bem como as relações com os fatores ambientais, proporcionando subsídios para que se possam distinguir os diferentes tipos de vegetação do bioma Caatinga e suas correlações. Tais levantamentos são atividades que visam obter informações qualitativas e quantitativas dos recursos florestais existentes em áreas pré-especificadas, imprescindíveis para administrá-las e para planejar sua utilização racional (Péllico Netto \& Brena, 1997).

No Brasil, país com dimensões continentais e uma grande carência de informações adequadas para a tomada de decisões sobre os problemas ambientais, o geoprocessamento apresenta um enorme potencial, quando baseado em tecnologias de custo relativamente baixo, em que o conhecimento seja adquirido localmente (Câmara \& Davis, 2001), sendo atualmente uma das ferramentas mais utilizadas nos inventários florestais.

Portanto, este trabalho teve como objetivo quantificar os remanescentes florestais do município de Teixeira, $\mathrm{PB}$, por meio de técnicas de geoprocessamento e inventário florestal.

\section{Material e métodos}

A região de estudo compreende o município de Teixeira, PB, localizado na mesorregião do Sertão Paraibano e na microrregião da Serra do Teixeira. O município possui área de $182 \mathrm{~km}^{2}$ (IBGE, 2010), com sede situada a $768 \mathrm{~m}$ de altitude, e coordenadas geográficas entre $37^{\circ} 08^{\prime} 22^{\prime \prime}$ a $37^{\circ} 25^{\prime} 33^{\prime \prime} \mathrm{W}$ e $07^{\circ} 11^{\prime} 10^{\prime \prime}$ a $07^{\circ} 21$ ' 23 ” S.

Segundo o Serviço Geológico do Brasil (CPRM) (Brasil, 2005), o município de Teixeira possui clima Aw'Quente úmido, com chuvas de verão e outono. De acordo com a divisão do Estado em regiões bioclimáticas, 
Teixeira se enquadra no bioclima 4ath-Tropical quente de seca acentuada, com sete a oito meses secos, temperatura média anual entre $23{ }^{\circ} \mathrm{C}$ e $24^{\circ} \mathrm{C}$ e pluviometria média anual de 714,6 mm, com distribuição irregular, sendo que $82 \%$ de seu total concentra-se em quatro meses, de janeiro a abril. O relevo varia de ondulado a fortemente ondulado, com cotas que variam de $630 \mathrm{~m}$, como ocorre na porção sudeste, até cotas mais elevadas e superiores a $750 \mathrm{~m}$, atingindo $960 \mathrm{~m}$, como acontece ao norte, nas serras do Teixeira. De acordo com Santos et al. (2007), os solos da região de Teixeira pertencem à classe de solos Litólicos Eutróficos, pouco desenvolvidos, com horizonte A fraco, textura média e muito rasa, e Cambissolos, associados a solos Litólicos, refletindo uma cobertura vegetal de caatinga hiperxerófila.

A atividade econômica mais importante do município de Teixeira é a agropecuária. $\mathrm{O}$ principal suporte da economia está concentrado no setor Primário, cuja participação situa-se na faixa de 25,1 a 50\% (Santos et al., 2007). Na agricultura, destacam-se as plantações de milho, feijão e mandioca. Na pecuária, a criação de bovinos e caprinos e, na avicultura, a criação de galináceos, com produção de ovos (Brasil, 2005).

Para o mapeamento da vegetação nativa, foram utilizadas imagens de satélites, que serviram de apoio para quantificação da vegetação, que foi dividida nas seguintes etapas: processamento digital das imagens, classificação temática e quantificação da vegetação. A imagem utilizada foi obtida do Satélite CBERS, (China-Brasil Earth Resources Satellite) referente ao mês de novembro de 2008, que foi processada e tratada com o auxílio do software IDRISI ${ }^{\circledR}$, versão 14.0. no Laboratório de Fotointerpretação do Centro de Saúde e Tecnologia Rural da Universidade Federal de Campina Grande, Patos, PB.

Os cálculos das áreas de vegetação foram efetuados por meio de mapas digitais, atualizando-se a vegetação de acordo com os diferentes estratos. Após a definição das áreas de cobertura vegetal e de ação antrópica, foi realizado inventário florestal, com uso de amostragem por parcelas, distribuídas sistematicamente, em um total de 40 unidades amostrais, com dimensões de $20 \mathrm{~m}$ x $20 \mathrm{~m}$, totalizando $16.000 \mathrm{~m}^{2}$ de área amostral da cobertura vegetal nativa. Foram estabelecidas distâncias entre parcelas de $1 \mathrm{~km}$ no sentido Norte-Sul e 2,5 km Leste-Oeste.
Cada árvore foi identificada pelo seu nome popular e científico. Foram amostrados todos os indivíduos inseridos nas parcelas, com diâmetro a $1,30 \mathrm{~m}$ do solo (DAP) maior ou igual a $10 \mathrm{~cm}$, medidos com o auxílio de uma fita métrica, e a altura total $(\mathrm{H})$, utilizando-se baliza graduada até $4 \mathrm{~m}$, sendo estimadas as alturas acima deste valor.

Para caracterizar a estrutura da vegetação, foram calculados, para cada espécie, os parâmetros fitossociológicos: densidade relativa; frequência relativa; dominância relativa; valor de importância (VI) e índice de diversidade de Shannon (H') (Felfili \& Rezende, 2003; Felfili et al., 2011).

Os cálculos dos parâmetros fitossociológicos e área basal foram feitos utilizando-se o software Mata Nativa ${ }^{\odot}$.

\section{Resultados e discussão}

Atualmente, a cobertura vegetal no município de Teixeira, PB corresponde a uma área de 11.760,1 ha, equivalente a $64,5 \%$ da área total do município. As áreas antropizadas, correspondem a 6.008,9 ha, o que representa 32,9\% (Figura 1). O restante, 506,5 ha, equivale às áreas urbanas, corpos d'água e rodovias que cortam o município. É importante salientar que não foram estimadas as estradas vicinais.

As áreas onde se observa maior devastação são aquelas próximas aos mananciais, como os açudes São Francisco, Poços e Sabonete, que se apresentam ocupadas por cultivo de hortaliças e culturas irrigadas, e aquelas utilizadas para agricultura de subsistência, principalmente em locais de relevo menos acidentado.

As regiões mais preservadas se encontram em áreas serranas, de difícil acesso e relevo acidentado, impróprias para agricultura, embora nestes locais ainda se encontrem vestígios de Agave sisalana, indicando que nestas áreas já ocorreu ação antrópica.

A vegetação arbórea amostrada foi de 4.911 indivíduos, pertencentes a 46 espécies e 24 famílias (Tabela 1), o que corresponde a 3.069 ind. ha-1.

Santana \& Souto (2006) encontraram o valor médio de 4.080 indivíduos.ha ${ }^{-1} \mathrm{em}$ estudo fitossociológico em caatinga, na Estação Ecológica do Seridó, RN, utilizando 30 parcelas de $20 \mathrm{~m}$ x $10 \mathrm{~m}$. 
Tabela 1. Relação florística das espécies arbustivo-arbóreas do município de Teixeira, PB.

\begin{tabular}{|c|c|c|}
\hline Família/Espécie & Nome Popular & Hábito \\
\hline \multicolumn{3}{|l|}{ Anacardiaceae } \\
\hline Myracrodruon urundeuva Allemão & Aroeira & Arbóreo \\
\hline \multicolumn{3}{|l|}{ Annonaceae } \\
\hline Rollinia leptopetala (R.E. Fries) Safford & Pinha-brava & Arbustivo \\
\hline \multicolumn{3}{|l|}{ Apocynaceae } \\
\hline Aspidosperma pyrifolium Mart. & Pereiro & Arbóreo \\
\hline Aspidosperma ulei Markgr. & Pitiá & Arbóreo \\
\hline \multicolumn{3}{|l|}{ Bignoniaceae } \\
\hline Tabebuia impetiginosa (Mart. ex DC.) Stdl. & Pau-d'arco & Arbóreo \\
\hline \multicolumn{3}{|l|}{ Boraginaceae } \\
\hline Cordia leucocephala Moric. & Maria-Preta & Arbóreo \\
\hline \multicolumn{3}{|l|}{ Burseraceae } \\
\hline Commiphora leptophloeos (Mart.) J. B. Gillet & Imburana & Arbóreo \\
\hline \multicolumn{3}{|l|}{ Caesalpiniaceae } \\
\hline Senna spectabilis (DC.) Irwin \& Barneby & Canafístula & Arbóreo \\
\hline Caesalpinia pyramidalis Tul. & Catingueira & Arbóreo \\
\hline Hymenaea courbaril $\mathrm{L}$. & Jatobá & Arbóreo \\
\hline Caesalpinia ferrea Mart. Ex Tul. & Jucá & Arbóreo \\
\hline Bauhinia cheilantha (Bong.) Stand. & Mororó & Arbóreo \\
\hline \multicolumn{3}{|l|}{ Capparaceae } \\
\hline Capparis flexuosa (L.) L & Feijão Feijão-bravo & Arbustivo \\
\hline \multicolumn{3}{|l|}{ Combretaceae } \\
\hline Combretum leprosum Mart. & Mofumbo & Arbustivo \\
\hline Thiloa glaucocarpa (Mart.) Eichl. & Sipaúba & Arbóreo \\
\hline \multicolumn{3}{|l|}{ Erythroxylaceae } \\
\hline Erythroxylum pauferrense T. Plowman & Coração-de-negro & Arbóreo \\
\hline \multicolumn{3}{|l|}{ Euphorbiaceae } \\
\hline Sapium sp. & Burra-leiteira & Arbóreo \\
\hline Acalypha multicalis Müll. Arg. & Catinga-branca & Arbóreo \\
\hline Manihot glaziovii Müll. Arg. & Maniçoba & Arbóreo \\
\hline Croton sonderianus Müll. Arg. & Marmeleiro & Arbóreo \\
\hline Jatropha molissima Müll. Arg. & Pinhão & Arbustivo \\
\hline \multicolumn{3}{|l|}{ Fabaceae } \\
\hline Pithecolobium polycephalum Benth. & Camundongo & Arbóreo \\
\hline Aeschynomene rudis Benth. & Canafistulazinha & Arbustivo \\
\hline Marchaerium cultratum Pittier & Espinho-de-Judeu & Arbóreo \\
\hline Indeterminada & Pau-de-serrote & Arbóreo \\
\hline Diplotropis purpurea (Rich.) Amsh. & Sucupira & Arbóreo \\
\hline \multicolumn{3}{|l|}{ Lauraceae } \\
\hline Ocotea cymbarum H. B. K. & Louro & Arbóreo \\
\hline \multicolumn{3}{|l|}{ Meliaceae } \\
\hline Cedrela fissilis Vell. & Cedro & Arbóreo \\
\hline \multicolumn{3}{|l|}{ Mimosaceae } \\
\hline Hymenolobium cericeum Ducke & Angelim-pedra & Arbóreo \\
\hline Anadenanthera colubrina (Vell.) Brenan & Angico & Arbóreo \\
\hline Acacia riparia Kunth & Calumbi & Arbustivo \\
\hline Acacia piauihenses Benth. & Espinheiro-branco & Arbóreo \\
\hline Mimosa malacocentra Mart. & Jiquiri & Arbóreo \\
\hline Piptadenia stipulacea (Benth.) Ducke & Jurema-branca & Arbóreo \\
\hline Mimosa tenuiflora (Willd.) Poir & Jurema-preta & Arbóreo \\
\hline Mimosa ophthalmocentra Mart. ex Benth. & Jurema-vermelha & arbóreo \\
\hline \multicolumn{3}{|l|}{ Myrtaceae } \\
\hline Eugenia cearensis Berg. & Goiaba-brava & Arbustivo \\
\hline
\end{tabular}




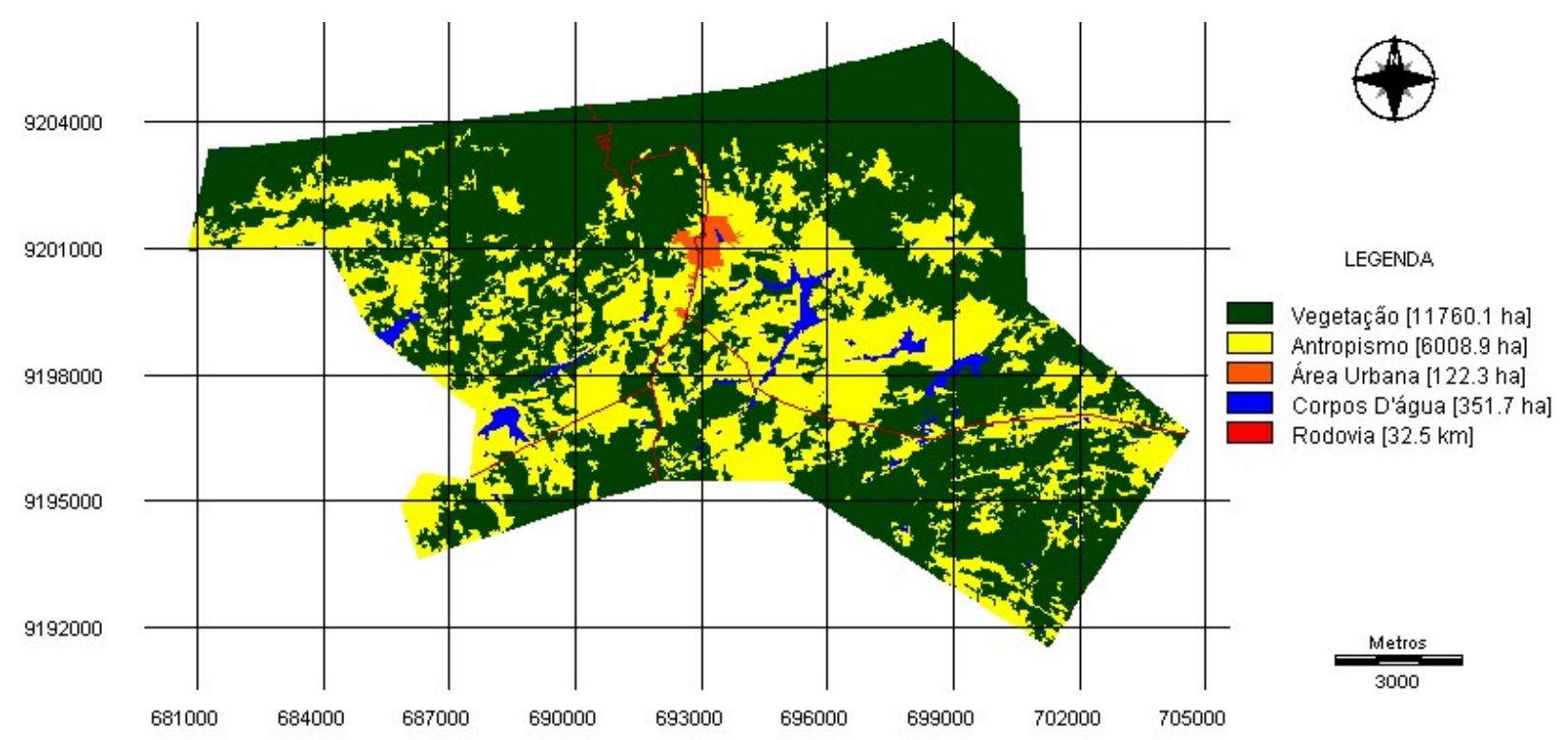

Figura 1: Cobertura vegetal do município de Teixeira, PB, em novembro de 2008.

As diferenças em número de espécies e famílias da Caatinga ficam evidentes quando se observa os resultados de levantamentos florísticos de diferentes autores. Silva (2005), estudando duas áreas de caatinga nos estados da Paraíba e Rio Grande do Norte, constatou a presença de 22 e 23 espécies, pertencentes a 14 e 19 famílias, respectivamente. Realizando trabalho semelhante, em um remanescente de caatinga no Seridó Paraibano, Fabricante \& Andrade (2007) encontraram 22 espécies pertencentes a oito famílias. Andrade et al. (2005) notificaram a presença de 16 espécies com oito famílias em uma área de caatinga no Cariri paraibano. Por outro lado, Costa et al. (2007) observaram número bem superior em número de espécies e famílias na Reserva Não Me Deixes, no estado do Ceará, contendo 300 ha de caatinga. Os autores relatam a ocorrência de 133 espécies, pertencentes a 47 famílias, sendo que a razão herbáceas/arbóreas foi de 4,1 .

Constata-se, portanto, que apesar de ter sido utilizada uma área amostral maior no presente estudo, o número de indivíduos por unidade de área foi menor. Porém, o número de espécies e famílias encontradas foram superiores ao relatado por outros autores (Andrade et al., 2005; Silva, 2005; Costa et al., 2007; Fabricante \& Andrade, 2007), podendo-se inferir que no município de Teixeira, PB, o remanescente estudado encontra-se em estado mais avançado de regeneração. Segundo Odum (1988), deve-se considerar que no processo de sucessão ecológica existe a tendência do meio passar de menos para mais complexo (número de espécies/famílias), com menor densidade por espécie (número de indivíduos/ espécies).

As famílias mais ricas em número de espécies arbustivoarbóreas, amostradas na vegetação do município de Teixeira, PB, foram Mimosaceae (8) Euphorbiaceae (5), Caesalpinaceae (5) e Fabaceae (5), que contribuíram com $2.151,1.278,395$ e 141 indivíduos, respectivamente, sendo também as mais representativas em número de indivíduos, representando, 43,8\%, 26,0\%, $8 \%$ e $2,9 \%$, respectivamente, de todos os indivíduos amostrados. As demais famílias contribuíram com duas ou uma espécie cada e representaram, em conjunto, $50 \%$ do total de espécies da área de estudo. Outras famílias, como Fabaceae, Anacardiaceae, Lauraceae, Erythroxylaceae, Apocynaceae, Capparaceae, Annonaceae, Boraginaceae, Bignoniaceae, Nyctaginaceae, Combretaceae, Celastraceae, Burseraceae, Verbenaceae, Olaceae, Meliaceae, Rhamnaceae, Rutaceae, Sapotaceae e Sapindaceae contribuíram, em conjunto, com 22,2\% do número de indivíduos amostrados (Figura 2).

Em trabalho realizado sobre composição florística em uma área de caatinga no semiárido paraibano por Araújo (2007), em Taperoá, PB, por Lacerda et al. (2005) e no sertão pernambucano, por Calixto Júnior \& Drumond (2011), foram observadas as mesmas três famílias botânicas contribuindo com o maior número de 
espécies, só que em ordem diferente. Em uma catinga arbórea em regeneração no Ceará, Braga \& Cavalcante (2007) observaram a família Mimosaceae como sendo a mais rica em número de indivíduos, seguida por Caesalpinaceae e Anacardinaceae. Esses resultados confirmam que estas famílias são as mais representativas em ambientes de caatinga.
Com relação ao número de indivíduos amostrados, Croton sonderianus respondeu por 36,04\% (Figura 3), confirmando a importância desta espécie na caatinga, conforme também observado por Santana \& Souto (2006). Além disso, áreas com grande ocorrência de $C$. sonderianus estão relacionadas com perturbações, pois esta espécie é sugerida como colonizadora de ambientes antropizados (Pinheiro \& Alves, 2007; Souza, 2009).

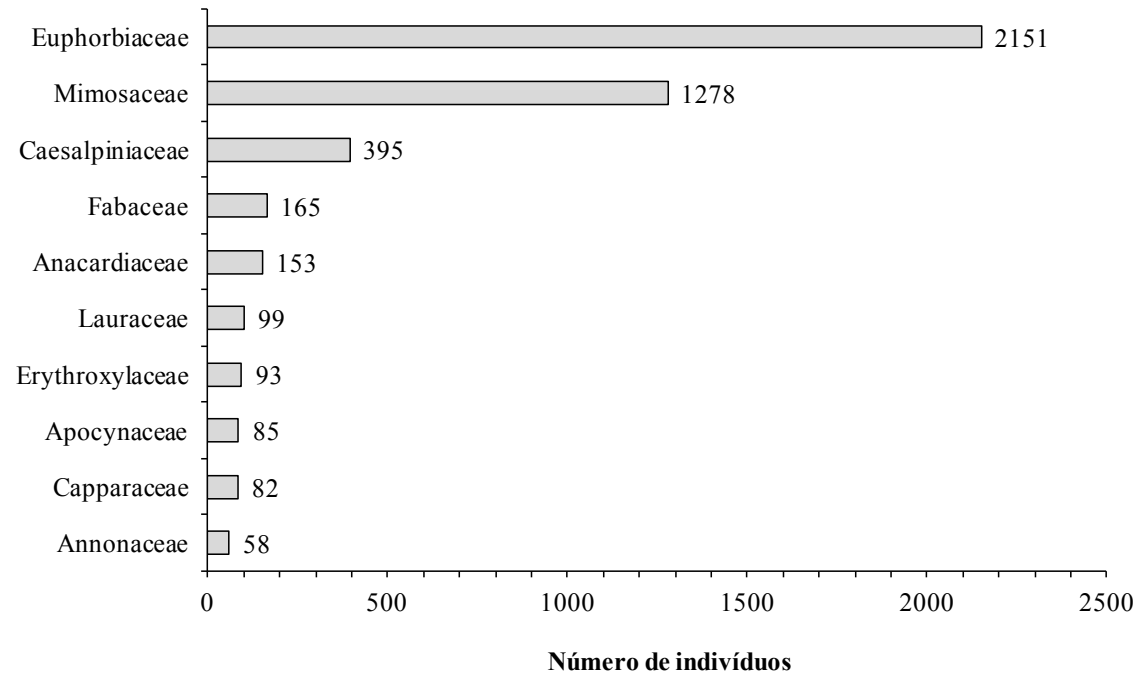

Figura 2. Relação das principais famílias botânicas do município de Teixeira, PB.

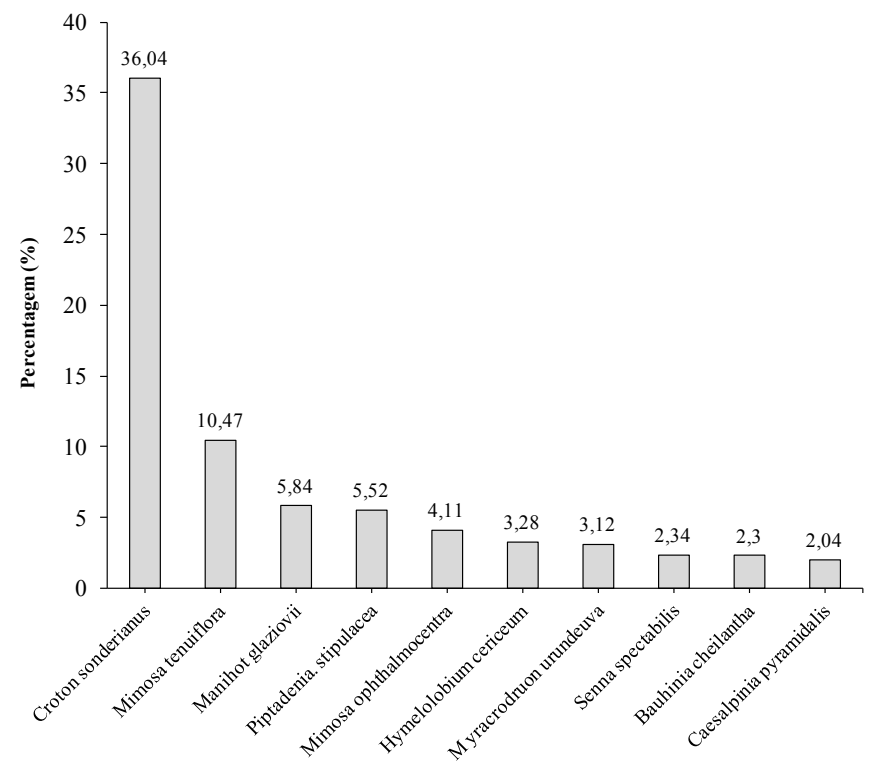

Figura 3. Percentual de indivíduos por espécie, município de Teixeira, PB. 
Espécies como Cedrela fissilis, Acacia piauhiensis e Caesalpinia ferrea ocorreram apenas em uma parcela cada; Zanthoxylum rhifolium e Thiloa glaucocarpa, em duas parcelas, todas localizadas em áreas de serra com difícil acesso, assim como Hymenolobium cericeum e Brumelia sertorium, indicando que a vegetação destes locais apresenta melhor grau de preservação, o que corrobora com a afirmação de Oliveira et al. (2009), que as áreas serranas guardam uma representativa parcela dos resquícios vegetacionais.

A espécie Aspidosperma pyrifolium foi observada apenas em altitudes inferiores a $332 \mathrm{~m}$, região de limite com o vale do Espinharas, onde esta espécie é encontrada com maior frequência, não sendo observada nas parcelas de maior altitude.

$\mathrm{O}$ índice de diversidade de Shannon-Weaver (H'), para a área estudada, foi de 2,69; o coeficiente de mistura de Jentsch (QM) foi de 1:104,49; o índice de dominância de Simpson (1-C), 0,99; e a equabilidade de Pielou (J), 0,70.

Segundo Felfili \& Rezende (2003), o índice de diversidade de Shannon assume que os indivíduos são amostrados aleatoriamente a partir de um conjunto infinitamente grande, e que todas as espécies estão representadas na amostra, baseando-se, portanto, na abundância proporcional das espécies, cujos valores situam-se, geralmente, entre 1,3 e 3,5, podendo alcançar $4,5 \mathrm{em}$ ambiente de floresta tropical. O valor encontrado neste estudo $(2,69)$ foi superior aos encontrados por Silva (2005) em áreas de caatinga na Estação Ecológica do Seridó (ESEC Seridó) no município de Serra Negra do Norte, RN e na Fazenda Oriente, município de Condado, $\mathrm{PB}$, que foram de 2,24 e 2,45 nats.ind ${ }^{-1}$, respectivamente, porém foi inferior ao encontrado por Alcoforado-Filho et al. (2003), em estudo realizado em Caruaru, PE (3,09 nats.ind $\left.{ }^{-1}\right)$.

O índice de dominância de Simpson indica a probabilidade de dois indivíduos quaisquer, retirados aleatoriamente de uma comunidade, pertencerem a diferentes espécies, dando mais peso à abundância das espécies e sendo menos sensível à riqueza (Felfili $\&$ Rezende, 2003). O valor calculado de (1-C) ocorre na escala de 0 a 1 , sendo que os valores próximos de 1 indicam menor diversidade (Scolforo et al., 2008). Neste estudo, o índice de Simpson $(0,99)$ também foi superior aos encontrados por Silva (2005), que não foram superiores a 0,88 , indicando que a área onde foi realizado este estudo apresenta uma menor diversidade.
O coeficiente de mistura de Jentsch $(\mathrm{QM})$ representa o número total de espécies encontradas no povoamento em relação ao número de indivíduos amostrados, de modo que quanto maior a mistura, ou seja, maior numerador, maior será a diversidade. Desse modo, no presente estudo o valor encontrado de 1:104,49 foi inferior aos encontrados em Serra Negra do Norte, RN e Condado, PE, (1:73,22 e 1:75,27, respectivamente) por Silva (2005), inferindo que a área estudada em Teixeiras, PB apresenta menor diversidade.

O índice de equabilidade de Pielou (J') é derivado do índice de diversidade de Shannon e permite representar a uniformidade da distribuição dos indivíduos entre as espécies existentes, como mencionado por (Scolforo et al., 2008). Seu valor apresenta uma amplitude de 0 (uniformidade mínima) a 1 (uniformidade máxima), indicando que a uniformidade encontrada no presente estudo $(0,70)$ é semelhante às encontradas por Silva (2005), de 0,71 e 0,72, respectivamente, para Serra Negra do Norte, RN e Condado, PE.

As espécies que mais contribuíram com número de indivíduos foram: Croton. sonderianus, com 1.770 indivíduos (1.106 indivíduos.ha-1); Mimosa tenuiflora, com 514 (321 indivíduos.ha ${ }^{-1}$ ); Manihot glaziovii, com 287 (179 indivíduos.ha-1); Piptadenia stipulacea, com 271 (59 indivíduos.ha ${ }^{-1}$ ); Mimosa ophthalmocentra, com 202 (126 indivíduos.ha-1 ${ }^{-1}$; Hymenolobium cericeum, com 171 (107 indivíduos.ha-1); Myracroduon urundeuva, com 153 (95 indivíduos.ha-1); Senna spectabillis, com 115 (72 indivíduos.ha ${ }^{-1}$ ); Bauhinia cheilantha, com 113 (71 indivíduos.ha-1) e Caesalpinia pyramidalis, com 100 (62 indivíduos.ha-1 ${ }^{-1}$.

Andrade et al. (2005), em uma área de caatinga no cariri paraibano, observaram a espécie Croton sonderianus como a de maior número de indivíduos, seguida de Caesalpinia pyramidalis, Aspidosperma pyrifolium e Jatropha mollissima. Silva (2005) também observou Croton sonderianus com o maior número de indivíduos em área de caatinga. Fabricante \& Andrade (2007) encontraram resultados semelhantes para as espécies mais abundantes em um remanescente de caatinga no seridó paraibano, observando 1.347,5 indivíduos.ha ${ }^{-1}$ de Croton sonderianus.

Das espécies amostradas, Croton sonderianus ocorreu em $92,5 \%$ das parcelas, ficando ausente apenas nos locais de difícil acesso, onde a vegetação encontra-se mais preservada. Outras espécies também apresentaram boa distribuição na área de estudo, apresentando frequência 
superior a 50\%, como é o caso de Piptadenia stipulacea, Mimosa ophthalmocentra, Manihot glaziovii e Mimosa tenuiflora, espécies consideradas pioneiras, presentes em áreas que já foram degradadas e se encontram em estágio médio de regeneração.
As dez espécies com maior valor de importância (VI) no município de Teixeira, PB foram: Croton sonderianus, Mimosa tenuiflora, Piptadenia stipulacea, Manihot glaziovii, Mimosa ophtalmocentra, Myracrodruon urundeuva, Anadenanthera colubrina, Caesalpinia pyramidalis, Bauhinia cheilantha e Senna spectabilis (Tabela 2).

Tabela 2. Parâmetros fitossociológicos das espécies amostradas no município de Teixeira, PB, ordenadas pelo valor de importância (VI).

\begin{tabular}{|c|c|c|c|c|c|c|c|c|c|}
\hline Espécie & DA & DR & FA & FR & DoA & DoR & $\mathrm{VC}$ & VI & VI (\%) \\
\hline Croton sonderianus & $1.106,250$ & 36,04 & 92,50 & 9,27 & 2,050 & 26,45 & 62,487 & 71,760 & 23,92 \\
\hline Mimosa tenuiflora & 321,250 & 10,47 & 57,50 & 5,76 & 1,046 & 13,49 & 23,960 & 29,725 & 9,91 \\
\hline Piptadenia stipulacea & 169,375 & 5,52 & 82,50 & 8,27 & 0,418 & 5,40 & 10,915 & 19,185 & 6,40 \\
\hline Manihot glaziovii & 179,375 & 5,84 & 62,50 & 6,27 & 0,539 & 6,96 & 12,803 & 19,069 & 6,36 \\
\hline Mimosa ophthalmocentra & 126,250 & 4,11 & 65,00 & 6,52 & 0,255 & 3,29 & 7,408 & 13,924 & 4,64 \\
\hline Myracrodruon urundeuva & 95,625 & 3,12 & 50,00 & 5,01 & 0,437 & 5,64 & 8,757 & 13,769 & 4,59 \\
\hline Anadenanthera colubrina & 35,000 & 1,14 & 25,00 & 2,51 & 0,440 & 5,68 & 6,823 & 9,329 & 3,11 \\
\hline Caesalpinia pyramidalis & 62,500 & 2,04 & 47,50 & 4,76 & 0,141 & 1,82 & 3,860 & 8,622 & 2,87 \\
\hline Bauhinia cheilantha & 70,625 & 2,30 & 45,00 & 4,51 & 0,107 & 1,37 & 3,675 & 8,186 & 2,73 \\
\hline Senna spectabilis & 71,875 & 2,34 & 22,50 & 2,26 & 0,222 & 2,87 & 5,209 & 7,464 & 2,49 \\
\hline Capparis flexuosa & 51,250 & 1,67 & 37,50 & 3,76 & 0,106 & 1,37 & 3,041 & 6,801 & 2,27 \\
\hline Ocotea cymbarum & 61,875 & 2,02 & 30,00 & 3,01 & 0,115 & 1,48 & 3,496 & 6,504 & 2,17 \\
\hline Hymenolobium cericeum & 100,625 & 3,28 & 2,50 & 0,25 & 0,201 & 2,59 & 5,866 & 6,117 & 2,04 \\
\hline Hymenaea courbaril & 41,250 & 1,34 & 7,50 & 0,75 & 0,242 & 3,12 & 4,463 & 5,215 & 1,74 \\
\hline Erythroxylum pauferrense & 58,125 & 1,89 & 12,50 & 1,25 & 0,158 & 2,04 & 3,935 & 5,188 & 1,73 \\
\hline Acalypha multicalis & 44,375 & 1,45 & 20,00 & 2,01 & 0,083 & 1,07 & 2,516 & 4,521 & 1,51 \\
\hline Commiphora leptophloeos & 11,875 & 0,39 & 25,00 & 2,51 & 0,094 & 1,22 & 1,605 & 4,112 & 1,37 \\
\hline Marchaerium cultratum & 48,125 & 1,57 & 10,00 & 1,00 & 0,103 & 1,33 & 2,900 & 3,903 & 1,30 \\
\hline Cordia leucocephala & 21,250 & 0,69 & 27,50 & 2,76 & 0,033 & 0,42 & 1,114 & 3,871 & 1,29 \\
\hline Tabebuia impetiginosa & 20,625 & 0,67 & 12,50 & 1,25 & 0,133 & 1,72 & 2,390 & 3,644 & 1,21 \\
\hline Guapira sp. & 19,375 & 0,63 & 22,50 & 2,26 & 0,053 & 0,68 & 1,309 & 3,564 & 1,19 \\
\hline Rollinia leptopetala & 36,250 & 1,18 & 15,00 & 1,50 & 0,063 & 0,81 & 1,992 & 3,495 & 1,17 \\
\hline Aspidosperma ulei & 32,500 & 1,06 & 17,50 & 1,75 & 0,049 & 0,63 & 1,687 & 3,442 & 1,15 \\
\hline Desconhecida & 28,125 & 0,92 & 17,50 & 1,75 & 0,051 & 0,65 & 1,570 & 3,324 & 1,11 \\
\hline D. guianensis & 33,125 & 1,08 & 12,50 & 1,25 & 0,077 & 1,00 & 2,079 & 3,332 & 1,11 \\
\hline Lantana camara & 10,625 & 0,35 & 25,00 & 2,51 & 0,015 & 0,19 & 0,535 & 3,041 & 1,01 \\
\hline Indeterminada & 15,625 & 0,51 & 20,00 & 2,01 & 0,028 & 0,36 & 0,870 & 2,875 & 0,96 \\
\hline Jatropha molissima & 10,000 & 0,33 & 20,00 & 2,01 & 0,022 & 0,29 & 0,613 & 2,618 & 0,87 \\
\hline Acacia riparia & 29,375 & 0,96 & 10,00 & 1,00 & 0,044 & 0,57 & 1,525 & 2,528 & 0,84 \\
\hline Mimosa malacocentra & 15,625 & 0,51 & 12,50 & 1,25 & 0,047 & 0,60 & 1,113 & 2,366 & 0,79 \\
\hline Aeschynomene rudis & 23,125 & 0,75 & 10,00 & 1,00 & 0,042 & 0,54 & 1,290 & 2,292 & 0,76 \\
\hline Combretum leprosum & 18,750 & 0,61 & 7,50 & 0,75 & 0,042 & 0,54 & 1,148 & 1,900 & 0,63 \\
\hline Aspidosperma pyrifolium & 20,625 & 0,67 & 2,50 & 0,25 & 0,075 & 0,97 & 1,640 & 1,891 & 0,63 \\
\hline Pithecolobium polycephalum & 16,250 & 0,53 & 7,50 & 0,75 & 0,034 & 0,44 & 0,970 & 1,722 & 0,57 \\
\hline Ximenia americana & 7,500 & 0,24 & 12,50 & 1,25 & 0,012 & 0,16 & 0,403 & 1,656 & 0,55 \\
\hline Thiloa glaucocarpa & 18,125 & 0,59 & 5,00 & 0,50 & 0,033 & 0,43 & 1,022 & 1,523 & 0,51 \\
\hline Cedrela fissilis & 6,250 & 0,20 & 2,50 & 0,25 & 0,081 & 1,04 & 1,245 & 1,495 & 0,50 \\
\hline Eugenia cearensis & 10,625 & 0,35 & 7,50 & 0,75 & 0,015 & 0,19 & 0,539 & 1,290 & 0,43 \\
\hline Ziziphus joazeiro & 5,625 & 0,18 & 7,50 & 0,75 & 0,016 & 0,21 & 0,391 & 1,143 & 0,38 \\
\hline Sapium sp. & 5,000 & 0,16 & 7,50 & 0,75 & 0,009 & 0,11 & 0,278 & 1,030 & 0,34 \\
\hline Zanthoxylum rhifolium & 4,375 & 0,14 & 5,00 & 0,50 & 0,010 & 0,13 & 0,274 & 0,776 & 0,26 \\
\hline Acacia piaihienses & 1,250 & 0,04 & 2,50 & 0,25 & 0,002 & 0,03 & 0,069 & 0,319 & 0,11 \\
\hline Lippia gracillis & 1,250 & 0,04 & 2,50 & 0,25 & 0,003 & 0,03 & 0,075 & 0,325 & 0,11 \\
\hline Talisia esculenta & 0,625 & 0,02 & 2,50 & 0,25 & 0,002 & 0,03 & 0,050 & 0,301 & 0,10 \\
\hline Brumelia sertorium & 0,625 & 0,02 & 2,50 & 0,25 & 0,001 & 0,02 & 0,036 & 0,286 & 0,10 \\
\hline Guettarda sericea & 0,625 & 0,02 & 2,50 & 0,25 & 0,001 & 0,01 & 0,028 & 0,279 & 0,09 \\
\hline Caesalpinia ferrea & 0,625 & 0,02 & 2,50 & 0,25 & 0,000 & 0,01 & 0,027 & 0,277 & 0,09 \\
\hline Total & $3.069,375$ & 100,0 & 997,5 & 100,0 & 7,750 & 100,0 & 200,0 & 300,0 & 100,0 \\
\hline
\end{tabular}

$\mathrm{DA}=$ densidade absoluta, $\mathrm{DR}=$ densidade relativa, $\mathrm{FA}=$ frequência absoluta, $\mathrm{FR}=$ frequência relativa, $\mathrm{DoA}=$ dominância absoluta, DoR $=$ dominância relativa, $\mathrm{VC}=$ valor de cobertura, $\mathrm{VI}=$ valor de importância e $\mathrm{VI} \%$ = valor de importância em porcentagem. 
Em trabalho realizado em uma área de caatinga do Seridó paraibano, Fabricante \& Andrade (2007) observaram a espécie favela (Cnidosculus phyllacanthus) (ausente na vegetação estudada no município de Teixeira, PB) com maior VI, seguida de C. pyramidalis, C. sonderianus e J. mollissima. Essas espécies representaram $72,92 \%$ da densidade relativa (DR), 54,14 da frequência relativa (FR), 72,97\% da dominância relativa (DoR), 72,95\% do valor de cobertura (VC) e $67,02 \%$ do VI. Os valores foram inferiores aos encontrados por Silva (2005) em duas áreas de caatinga do Rio Grande do Norte e Paraíba.

A maioria dos indivíduos foi representada no estrato médio $(4,05 \leq \mathrm{H}<6,16)$, com 3.740 indivíduos, 897 no estrato inferior $(\mathrm{H}<4,05)$ e 544 no estrato superior $(\mathrm{H} \geq$ $6,16)$. A altura mínima observada foi de $2 \mathrm{~m}$, a máxima foi de $10,5 \mathrm{~m}$ e a altura média de 5,0 $\mathrm{m}$. As espécies que apresentaram maior número de indivíduos com altura superior a 6,16 m foram Myracrodruon urundeuva, Anadenanthera colubrina, Tabebuia impetiginosa e Cedrela fissilis.
A altura média da vegetação amostrada no município de Teixeira, PB foi inferior à encontrada por Braga $\&$ Cavalcante (2007), em um fragmento de caatinga arbórea em regeneração no Ceará, que foi de $7,73 \mathrm{~m}$. A altura média de 5,0 $\mathrm{m}$ foi fortemente influenciada pelo grande número de indivíduos de Croton sonderianus, que apresentou uma grande frequência no estrato 4,05 e $6,16 \mathrm{~m}$ de altura, em um total de 1.192 , o equivalente a 745 indivíduos. ha ${ }^{-1}$.

Rodal et al. (2008), analisando a estrutura de caatinga do sertão central de Pernambuco, encontraram um valor médio para altura de $2,37 \mathrm{~m}$, inferior ao do presente estudo, porém com uma altura máxima de $12 \mathrm{~m}$.

A área basal estimada foi de $7,75 \mathrm{~m}^{2} \cdot \mathrm{ha}^{-1}$ (Tabela 3). As espécies que apresentaram maior área basal foram Croton sonderianus, Mimosa tenuiflora, Manihot glaziovii, Anadenanthera colubrina, Myracrodruon urundeuva, Piptadenia stipulacea, Mimosa ophthalmocentra, Hymenaea courbaril, Senna spectabilis e Hymenolobium cericeum.

Tabela 3. Distribuição da área basal por espécie, município de Teixeira, PB.

\begin{tabular}{lccc}
\hline \multicolumn{1}{c}{ Espécies } & $\mathbf{N}$ & $\mathbf{A B}\left(\mathbf{m}^{\mathbf{2}}\right)$ & $\mathbf{A B} \mathbf{( m}^{\mathbf{2}} \mathbf{. h} \mathbf{a}^{-\mathbf{1}} \mathbf{)}$ \\
\hline Croton sonderianus & 1.770 & 3,2793 & 2,0496 \\
\hline Mimosa tenuiflora & 514 & 1,6733 & 1,0458 \\
\hline Piptadenia stipulacea & 287 & 0,8630 & 0,5394 \\
\hline Manihot glaziovii & 56 & 0,7047 & 0,4404 \\
\hline Mimosa ophthalmocentra & 153 & 0,6996 & 0,4373 \\
\hline Hymenolobium cericeum & 271 & 0,6692 & 0,4183 \\
\hline Senna spectabilis & 202 & 0,4086 & 0,2554 \\
\hline Myracrodruon.urundeuva & 66 & 0,3868 & 0,2418 \\
\hline Bauhinia cheilantha & 115 & 0,3555 & 0,2222 \\
\hline Erythroxylum pauferrense & 161 & 0,3209 & 0,2006 \\
\hline Caesalpinia pyramidalis & 93 & 0,2531 & 0,1582 \\
\hline Ocotea cymbarum & 100 & 0,2261 & 0,1413 \\
\hline Marchaerium cultratum & 33 & 0,2131 & 0,1332 \\
Capparis flexuosa & 99 & 0,1836 & 0,1148 \\
\hline Acalypha multicalis & 113 & 0,1704 & 0,1065 \\
\hline Rollinia leptopetala & 82 & 0,1701 & 0,1063 \\
\hline Hymenaea courbaril & 77 & 0,1652 & 0,1033 \\
\hline Aspidosperma ulei & 19 & 0,1511 & 0,0944 \\
\hline D. guianensis & 71 & 0,1327 & 0,0829 \\
\hline Acacia riparia & 10 & 0,1291 & 0,0807 \\
\hline Desconhecida & 53 & 0,1240 & 0,0775 \\
\hline Combretum leprosum & 33 & 0,1201 & 0,0751 \\
\hline Cordia leucocephala & 58 & 0,1005 & 0,0628 \\
\hline Anadenanthera colubrina & 31 & 0,0840 & 0,0506 \\
\hline Guapira sp. & 45 & 0,0810 & \\
\hline & & & \\
\hline
\end{tabular}


Tabela 3. continuação

\begin{tabular}{|c|c|c|c|}
\hline Espécies & $\mathbf{N}$ & $\mathbf{A B}\left(\mathbf{m}^{2}\right)$ & $\mathrm{AB}\left(\mathrm{m}^{2} \cdot \mathrm{ha}^{-1}\right)$ \\
\hline Indeterminada & 52 & 0,0779 & 0,0487 \\
\hline Thiloa glaucocarpa & 25 & 0,0749 & 0,0468 \\
\hline Pithecolobium polycephalum & 47 & 0,0704 & 0,0440 \\
\hline Mimosa malacocentra & 30 & 0,0666 & 0,0416 \\
\hline Tabebuia impetiginosa & 37 & 0,0665 & 0,0416 \\
\hline Aspidosperma pyrifolium & 26 & 0,0547 & 0,0342 \\
\hline Commiphora leptophloeos & 29 & 0,0535 & 0,0334 \\
\hline Lantana camara & 34 & 0,0522 & 0,0326 \\
\hline Eugenia cearensis & 25 & 0,0447 & 0,0279 \\
\hline Aeschynomene rudis & 16 & 0,0356 & 0,0223 \\
\hline Jatropha molissima & 9 & 0,0258 & 0,0161 \\
\hline Ximenia americana & 17 & 0,0239 & 0,0149 \\
\hline Ziziphus joazeiro & 17 & 0,0234 & 0,0146 \\
\hline Sapium sp. & 12 & 0,0197 & 0,0123 \\
\hline Zanthoxylum rhifolium & 7 & 0,0163 & 0,0102 \\
\hline Cedrela fissilis & 8 & 0,0142 & 0,0089 \\
\hline Acacia piauihienses & 2 & 0,0042 & 0,0026 \\
\hline Guettarda sericea & 1 & 0,0037 & 0,0023 \\
\hline Talisia esculenta & 2 & 0,0035 & 0,0022 \\
\hline Brumelia sertorium & 1 & 0,0019 & 0,0012 \\
\hline Lippia gracillis & 1 & 0,0010 & 0,0006 \\
\hline Caesalpinia ferrea & 1 & 0,0008 & 0,0005 \\
\hline Total & 4.911 & 12,4010 & 7,7503 \\
\hline
\end{tabular}

O volume cilíndrico total estimado foi de 44,89 $\mathrm{m}^{3} \cdot \mathrm{ha}^{-1}$, o que equivale a um volume real de $40,4 \mathrm{~m}^{3} \cdot \mathrm{ha}^{-1} \mathrm{e}$ a um volume estéreo de 137,37 st.ha $^{-1}$, sendo que o maior volume encontrado foi para classe diamétrica entre 3,0 e $6,0 \mathrm{~cm}\left(17,3294 \mathrm{~m}^{3} \cdot \mathrm{ha}^{-1}\right)$, decrescendo de acordo com o aumento das classes diamétricas. Vale salientar que na classe de 24,0 a $27,0 \mathrm{~cm}$ não foi observado nenhum indivíduo (Tabela 4).

Tabela 4. Distribuição volumétrica por classe de diâmetro e por hectare, município de Teixeira, PB.

\begin{tabular}{cccc}
\hline $\begin{array}{c}\text { Centro de classes } \\
\text { de DAP }(\mathbf{c m})\end{array}$ & $\begin{array}{c}\text { Volume cilíndrico } \\
\left(\mathbf{m}^{3} \cdot \mathbf{h a}^{-1}\right)\end{array}$ & $\begin{array}{c}\text { Volume } \\
\text { real } \\
\left(\mathbf{m}^{3} \cdot \mathbf{h a}^{-1}\right)\end{array}$ & $\begin{array}{c}\text { Volume } \\
\text { estéreo } \\
\left(\mathbf{s t . h a}^{-1}\right)\end{array}$ \\
\hline $3,0-6,0$ & 17,3294 & 15,5965 & 53,0280 \\
$6,0-9,0$ & 12,0687 & 10,8618 & 36,9302 \\
$9,0-12,0$ & 5,2880 & 4,7592 & 16,1813 \\
$12,0-15,0$ & 3,0874 & 2,7787 & 9,4474 \\
$15,0-18,0$ & 2,1279 & 1,9151 & 6,5114 \\
$18,0-21,0$ & 0,9280 & 0,8352 & 2,8397 \\
$21,0-24,0$ & 2,5585 & 2,3027 & 7,8290 \\
$24,0-27,0$ & 0,0000 & 0,0000 & 0,0000 \\
$27,0-30,0$ & 0,7024 & 0,6322 & 2,1493 \\
$30,0-33,0$ & 0,8022 & 0,7220 & 2,4547 \\
\hline Total & $\mathbf{4 4 , 8 9 2 5}$ & $\mathbf{4 0 , 4 0 3 3}$ & $\mathbf{1 3 7 , 3 7 1 1}$ \\
\hline
\end{tabular}

O valor obtido para volume total foi superior aos observados por Silva (2005), que encontrou $22,95 \mathrm{~m}^{3} \cdot \mathrm{ha}^{-1}$ e $32,73 \mathrm{~m}^{3} \cdot$ ha $^{-1}$, na ESEC Seridó, Serra Negra do Norte, RN e Fazenda Oriente, Condado, PB, respectivamente.

Rodal et al. (1998), em um refúgio vegetacional no município de Buíque, PE, encontraram resultados semelhantes para distribuição diamétrica, observando que ocorreu a maior concentração de indivíduos na classe de 3 a $6 \mathrm{~cm}$ de diâmetro.

O maior diâmetro observado foi de $32 \mathrm{~cm}$, em um indivíduo de Tabebuia impetiginosa, com 9,0 m de altura. Araújo (2007) encontrou $68 \mathrm{~cm}$ como diâmetro máximo, em um indivíduo morto de Anadenanthera colubrina, e 10,5 m como altura máxima, em um indivíduo de Hymenaea courbaril.

As espécies que apresentaram maior volume foram Croton sonderianus, por ser a espécie com maior frequência de indivíduos, seguida por Mimosa tenuiflora, Anadenanthera colubrina, Myracrodruon urundeuva, Manihot glaziovii, Piptadenia stipulacea, Hymenaea courbaril, Senna spectabilis, Hymenolobium cericeum e Mimosa ophthalmocentra. 


\section{Conclusões}

O município de Teixeira apresenta áreas mais preservadas nas regiões serranas, de difícil acesso.

As famílias que apresentaram maior número de indivíduos foram Euphorbiaceae, Mimosaceae e Caesalpinaceae, sendo Croton sonderianus a espécie com maior número de indivíduos, seguido de Mimosa tenuiflora, Manihot glaziovii e Piptadenia stipulacea. Essas quatro foram as espécies com maior valor de importância, com destaque também para Croton sonderianus.

Croton sonderianus foi a espécie que obteve maior área basal, assim como maior volume, sendo que as parcelas com maiores áreas basais se encontram em áreas de difícil acesso.

\section{Referências}

ALCOFORADO-FILHO, F. G.; SAMPAIO, E. V. de S. B.; RODAL, M. de J. N. Florística e fitossociologia de um remanescente de vegetação caducifólia espinhosa arbórea em Caruaru, Pernambuco. Acta Botanica Brasilica, Feira de Santana, v.17, n. 2, p. 287-303, 2003. DOI: 10.1590/S0102-33062003000200011

ANDRADE, L. A. de; PEREIRA, I. M.; LEITE, U. T.; BARBOSA, M. R. V. Análise da cobertura de duas fitofisionomias de caatinga, com diferentes históricos de uso, no município de São João do Cariri, Estado da Paraíba. Cerne, Lavras, v. 11, n. 3, p. 253-262, 2005.

ARAÚJO, L. V. C. de. Composição florística, fitossociologia e influência dos solos da estrutura da vegetação em uma área de caatinga no semi-árido paraibano. 2007. 121 f. Tese (Doutorado em Agronomia) - Universidade Federal da Paraíba, Areia.

BRAGA, E. P.; CAVAlCANTE, A. de M. B. Florística e fitossociologia de um fragmento de caatinga arbórea em regeneração no Ceará. In: CONGRESSO DE ECOLOGIA DO BRASIL, 8., 2007, Caxambu. Ecologia no tempo de mudanças globais: programas e anais. Caxambu: SEB, 2007. CD-ROM.

BRASIL. Ministério de Minas e Energia. Serviço Geológico do Brasil. Projeto Cadastro de fontes de abastecimento por água subterrânea: Paraíba: diagnóstico do município de Teixeira Recife: CPRM/PRODEEM, 2005. 19 p.

CALIXTO JÚNIOR, J. T.; DRUMOND, M. A. Estrutura fitossociológica de um fragmento de caatinga sensu stricto 30 anos após corte raso, Petrolina-PE, Brasil. Revista Caatinga, Mossoró, v. 24, n. 2, p. 67-74, 2011.

CÂMARA, G.; DAVIS, C. Apresentação. In: CÂMARA, G.; DAVIS, C.; MONTEIRO, A. M. V. (Org.).Introdução à ciência da geoinformação: Por que Geoprocessamento? Cap. 1. São Paulo: INPE, 2001. 345 p. Disponível em: <http://www.dpi.inpe.br/ gilberto/ livro/introd.> Acesso em 10 abr. 2013.
COSTA, R. C. da; ARAÚJO, F. S. de; LIMA-VERDE, L. W. Flora and life-form spectrum in an area of deciduous thorn woodland (caatinga) in northeastern, Brazil. Journal of Arid Environments, v. 68 , p. 237-247, 2007. DOI: 10.1016/j.jaridenv.2006.06.003

FABRICANTE, J. R.; ANDRADE, L. A. Análise estrutural de um remanescente de Caatinga no Seridó Paraibano. Oecologia Brasiliensis, Rio de Janeiro, v.11, n. 3, p. 341-349, 2007.

FELFILI, J. M.; EISENLOHR, P. V.; MELO, M. M. F. de; ANDRADE, L. A. de; MEIRA-NETO, J. A. A. (Org.) Fitossociologia no Brasil: métodos e estudos de casos: volume 1. Viçosa, MG: Ed. da UFV, 2011. $556 \mathrm{p}$.

FELFILI, J. M.; REZENDE, R. P. Conceitos e métodos em fitossociologia. Brasília, DF: Universidade de Brasília, Departamento de Engenharia Florestal, 2003. 68 p. (Comunicações técnicas florestais, v. 5, n. 1).

FRANCA-ROCHA, W.; SILVA, A. de B.; NOLASCO, M. C.; LOBÃO, J.; BRITTO, D.; CHAVES, J. M.; ROCHA, C. C. da. Levantamento da cobertura vegetal e do uso do solo do Bioma Caatinga. In: SIMPÓSIO BRASILEIRO DE SENSORIAMENTO REMOTO, 13., 2007, Florianópolis. Anais... Florianópolis: INPE; SELPER, 2007. p. 2629-2636.

IBGE. Cidades. Rio de Janeiro, 2010.

LACERDA, A. V. de; NORDI, N.; BARBOSA, F. M.; WATANABE, T. Levantamento florístico do componente arbustivo-arbóreo da vegetação ciliar da bacia do rio Taperoá, PB, Brasil. Acta Botanica Brasilica, Feira de Santana, v. 19, n. 3, p. 647-656, 2005. DOI: 10.1590/S0102-33062005000300027

LEAL, R. D. K.; MACIEL, L. V. B.; PEREIRA, J. L. F.; AVELINO, M. C. S.; ROCHA, L. M. Conservação na caatinga: em que pé estamos? In: CONGRESSO DE ECOLOGIA DO BRASIL, 8., 2007, Caxambu. Ecologia no tempo de mudanças globais: programas e anais. Caxambu: SEB, 2007. CD-ROM

LEAL, I. R.; SILVA, J. M. C. da; TAVARELLI, M.; LACHER JUNIOR, T. E. Mudando o curso da conservação da biodiversidade na Caatinga do Nordeste do Brasil. Megadiversidade, Belo Horizonte, v. 1, n. 1, 2005.

MAIA, G. N. Caatinga: árvores e arbustos e suas utilidades. São Paulo: D \& Z, 2004. 413 p.

MONTEIRO, E. R.; MANGOLIN, C. A.; NEVES, A. F. das; ORASMO, G. R.; SILVA, J. G. M. da; MACHADO, M. F. P. S. Genetic diversity and structure of populations in Pilosocereus gounellei (F.A.C.Weber ex K.Schum.) (Cactaceae) in the Caatinga biome as revealed by heterologous microsatellite primers. Biochemical Systematics and Ecology, Oxford, v. 58, n. 2, p. 7-12, 2015. DOI: $10.1016 /$ j.bse.2014.10.006

ODUM, E. Ecologia. Rio de Janeiro: Guanabara Koogan, 1988. $434 \mathrm{p}$.

OLIVEIRA, P. T. B.; TROVÃO, D. M. B. M.; CARVALHO, E. C. D.; SOUZA, B. C.; FERREIRA, L. M. R. Florística e fitossociologia de quatro remanescentes vegetacionais em áreas de serra no cariri paraibano. Revista Caatinga, Mossoró, v. 22, n. 4, p. 169-178, 2009.

PÉLLICO-NETTO, S.; BRENA, D. A. Inventário Florestal. Curitiba, 1997. 316 p.

Pesq. flor. bras., Colombo, v. 35, n. 82, p. 89-100, abr.jun. 2015 
PINHEIRO, K.; ALVES, M. Espécies arbóreas de uma área de Caatinga no sertão de Pernambuco, Brasil: dados preliminares. Revista Brasileira de Biociências, Porto Alegre, v. 5, n. 2, p. 426428, 2007.

RAMALHO, C. I.; ANDRADE, A. P. de; FÉLIX, L. P.; LACERDA, A. V. de; MARACAJÁ, P. B. Flora arbóreo-arbustiva em áreas de Caatinga no Semi-Árido baiano, Brasil. Revista Caatinga, Mossoró, v. 22, n. 3, p. 182-190, 2009.

RODAL, M. J. N.; COSTA, K. C. C.; SILVA, A. C. B. e. Estrutura da vegetação caducifólia espinhosa (Caatinga) de uma área do sertão central de Pernambuco. Hoehnea, São Paulo, v. 35, n. 2, p. 209-217, 2008. DOI: $10.1590 / \mathrm{S} 2236-89062008000200004$

RODAL, M. J. N.; SILVA, A. C. B. L. E.; CARVALHO, K. C. B. Repartição espacial de comunidades de plantas lenhosas no semiárido do nordeste do Brasil. In: CONGRESSO DE ECOLOGIA DO BRASIL, 7., 2003, Fortaleza. Anais. Fortaleza: [s.n.] 2003.

RODAL, M. J. N.; ANDRADE, V. de A.; SALES, M. F.; GOMES, A. P. S. Fitossociologia do componente lenhoso de um refúgio vegetacional no município de Buíque, Pernambuco. Revista Brasileira de Biologia, São Carlos, v. 58, n. 3, p. 517-526, 1998. DOI: $10.1590 / \mathrm{S} 0034-71081998000300017$
SANTANA, J. A. da S.; SOUTO, J. S. Diversidade e estrutura fitossociológica da Caatinga na Estação Ecológica do Seridó-RN. Revista de Biologia e Ciências da Terra, João Pessoa, v. 6, n. 2, p. 232-242, 2006.

SANTOS, J. Y. G. dos; CUNHA, T. B.; VIANNA, P. C. G.; TRAVASSOS, I. S. A incidência de casos de câncer no município de Teixeira-PB e a sua relação com a possível contaminação das águas do açude São Francisco II por agrotóxico. In: SEMAGEO: Semana da Geografia da UFPB, 4, 2007, João Pessoa. Anais... João Pessoa: UFPB, 2007.

SCOLFORO, J. R.; OLIVEIRA, A. D.; FERRAZ FILHO, A. C.; MELLO, J. M. Diversidade, equabilidade e similaridade no domínio da caatinga. In: MELLO, J. M.; SCOLFORO, J. R.; CARVALHO, L. M. T. (Ed.). Inventário Florestal de Minas Gerais: Floresta Estacional Decidual: florística, estrutura, similaridade, distribuição diamétrica e de altura, volumetria, tendências de crescimento e manejo florestal. Lavras: UFLA, 2008. p.118-133. Disponível em: $<$ http://www.inventarioflorestal.mg.gov.br/ publicacoes/decidua/ capitulo06.pdf $>$. Acesso em: 18 mar. 2015.

SILVA, J. A. Fitossociologia e relações alométricas em caatinga nos Estados da Paraíba e Rio Grande do Norte. 2005. 93 f. Tese (Doutorado em Ciência Florestal) - Universidade Federal de Viçosa, Viçosa, MG. 